\title{
The aftermath of SARS-CoV-2 in NICU: saving or checking accounts? Projected cost-effectiveness analysis
}

\author{
Alfonso Galderisi ${ }^{1}$ (D) Elisabetta Lolli ${ }^{1} \cdot$ Maria Elena Cavicchiolo ${ }^{1} \cdot$ Luca Bonadies $^{1} \cdot$ Daniele Trevisanuto $^{1}$. \\ Eugenio Baraldi ${ }^{1}$
}

Received: 20 August 2020 / Revised: 13 November 2020 / Accepted: 20 November 2020 / Published online: 7 January 2021

(C) Springer-Verlag GmbH Germany, part of Springer Nature 2021

\begin{abstract}
In the aftermath of the SARS-CoV-2 pandemic, we revised the cost-effectiveness of the exploited interventions in neonatal intensive care unit, to redefine future strategies for hospital management. Costs were revised with respect to the lockdown R0 or under different $\mathrm{R} 0$ scenarios to estimate the cost-effectiveness of the screening program adopted. Weekly nasopharyngeal swabs for parents, neonates, and personnel were the major cost during the pandemic, although they effectively reduced the number of cases in our unit.

Conclusion: Parents and healthcare personnel testing appears to be an effective strategy due to the high number of contact they have within the hospital environment and outside, able to minimize the cases within our unit.

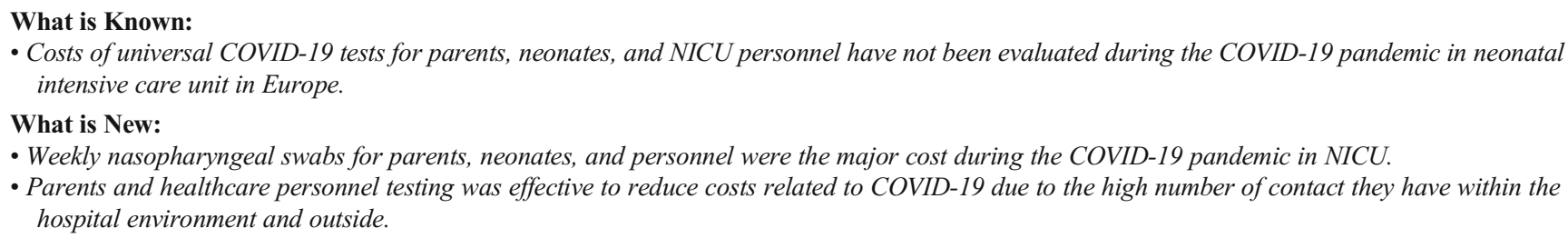

Keywords Preterm infant $\cdot$ Neonatal intensive care unit $\cdot$ SARS-CoV-2 $\cdot$ COVID-19 $\cdot$ Cost-effectiveness $\cdot$ Screening

\author{
Communicated by Daniele De Luca \\ Alfonso Galderisi \\ alfonso.galderisi@unipd.it \\ Elisabetta Lolli \\ elisabetta.lolli@aopd.veneto.it \\ Maria Elena Cavicchiolo \\ marialena.cavicchio@aopd.veneto.it \\ Luca Bonadies \\ luca.bonadies@aopd.veneto.it \\ Daniele Trevisanuto \\ daniele.trevisanuto@unipd.it \\ Eugenio Baraldi \\ eugenio.baraldi@unipd.it \\ 1 Department of Women's and Children's Health, University Hospital \\ of Padova, Via N. Giustiniani, 3, 35128 Padova, Italy
}

\author{
Abbreviations \\ NICU Neonatal intensive care unit
}

\section{Introduction}

In the aftermath of the SARS-CoV-2 pandemic, it comes the time to evaluate the cost-effectiveness of the exploited interventions to redefine future strategies for hospital management.

Although the SARS-CoV-2 impact in neonatal care has been clinically minimal, the pandemic has heavily affected the work organization in neonatal critical care setting [1].

Neonatal infection seems to be rare and acquired later during breastfeeding or neonatal care [2], and the respiratory outcomes have been shown in small cohorts to be favorable [3]; however, vertical neonatal transmission has been documented and needs to be accounted during delivery room interventions and early neonatal care [4]. 
We fall short of evidence about the contagious rate among neonatal intensive care unit (NICU) personnel as well as from neonate to personnel [5]. As for all the pediatric healthcare facilities, the access of families has to be limited and parents/ caregivers need to be trained to the use of personal protective equipment (PPE). The practice of testing the parents as well as the personnel is still largely limited due to the costs and the facility available to this task.

Herein, we describe the cost-effectiveness of systematic testing and individual protection of medical and non-medical personnel, patients, and their parents in a neonatal intensive care unit hub in north-eastern Italy.

\section{Methods}

Setting The neonatal intensive care unit of the University Hospital of Padova is the hub center for north-eastern Italy with $\sim 3000$ admissions/year and 100 neonates/year with very low birth weight. Padova NICU is a facility with a total of 35 cradles, 20 for intensive care, three open spaces with six beds each, and two isolation rooms with negative pressure. The personnel consist of 12 neonatologists, 15 residents, 56 nurses, and four non-medical assistants.

Measurements during SARS-CoV-2 Parents' access has not been restricted, with weekly swab test, daily temperature measurements, and PPE before the admittance after a specific training for the use of PPE in NICU area. Personnel have been tested weekly, with daily monitoring of temperature. Each neonate admitted to NICU has been tested with RT-PCR for
SARS-CoV-2 (collected by nasopharyngeal swab), regardless of maternal status.

Cost analysis The first three trimesters of 2019 and the first three trimesters of 2020 were compared for the descriptive cost analysis. The projection of the expected costs under different scenarios accounted for the actual costs (first trimester of 2020) with the number of cases presented as compared with the expected costs under the scenario of one or more key person involved. The effectiveness was, furtherly, evaluated assuming an R0 (number of people expected to be infected by a single case) from 0.5 to 4.5 (4.5 was the peak R0 reported in the region and 2.68 the maximum R0 from Wuhan area).

Salaries were considered "savings" when one or more healthcare providers (HCPs) were projected as infected and quarantined for 15 days with necessity for a personnel unit for replacement.

Contacts have been described according to the standardized procedures of our setting; therefore, each parent is exposed to the room nurse and to one resident, one attending, and one non-medical HCP, plus her/his own baby (Fig. 1).

Estimated savings-costs difference was estimated as follows:

Savings-costs $=$ average salary for the number estimated contacts - costs of prevention campaign.

The average salary was calculated for the minimum absence of 14 days prescribed to the asymptomatic cases. The cost of prevention campaign was the cost of daily PPE + weekly nasopharyngeal swab.

The savings-costs difference was estimated under three different scenarios: (1) screening for HCP and neonates (no parents);
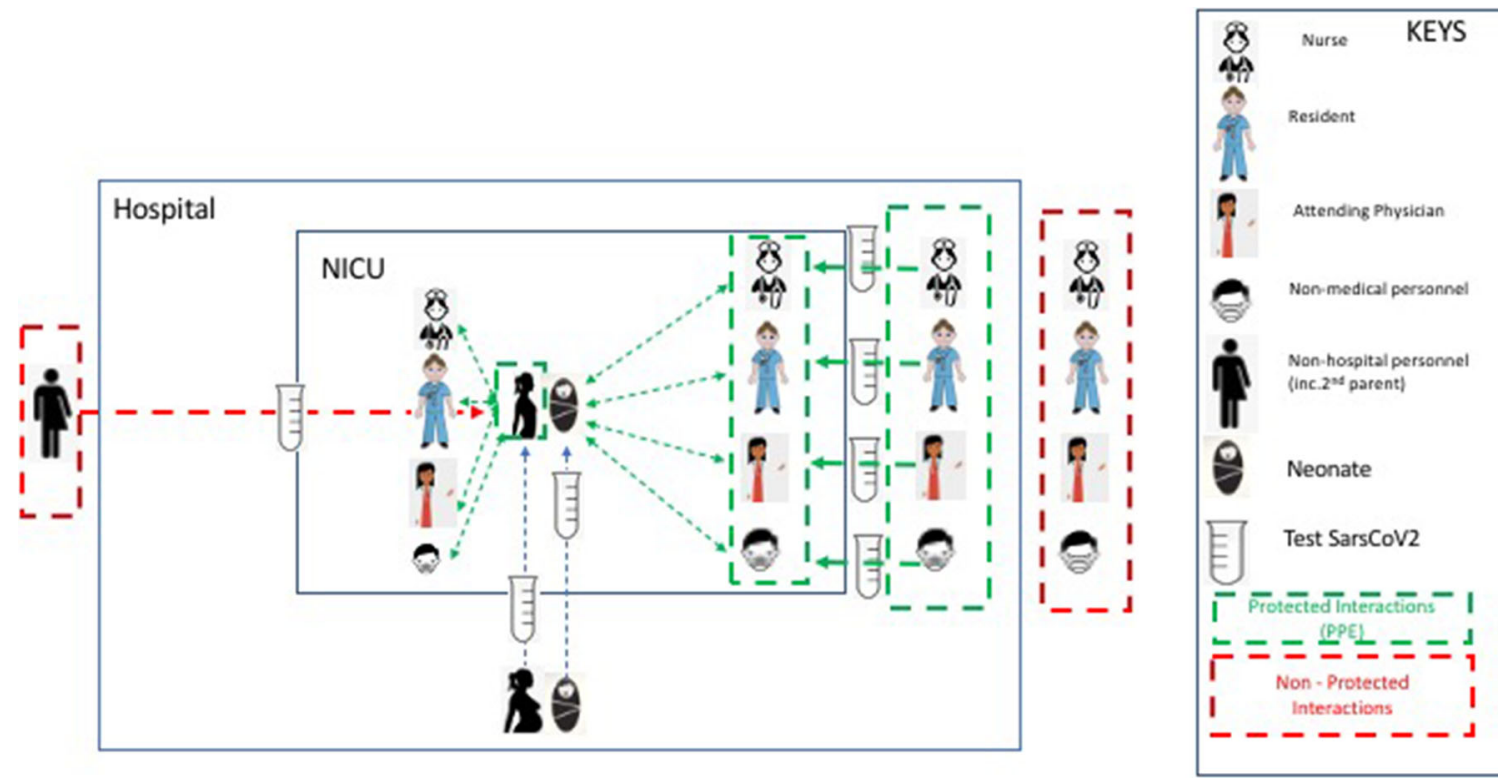

Fig. 1 Contacts within neonatal intensive care unit according to the work organization. HCP, healthcare personnel 


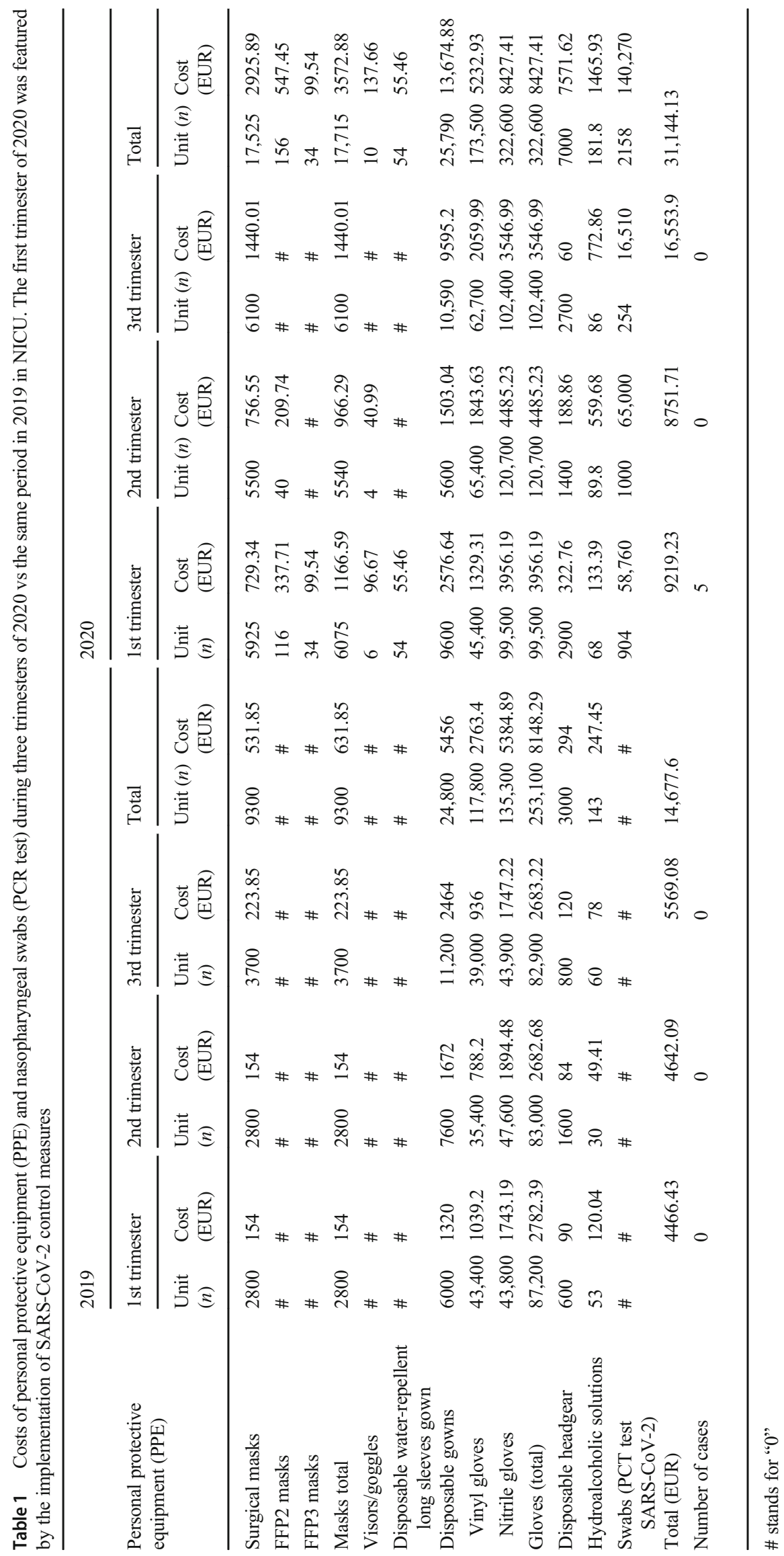


(2) screening for neonates and parents (no personnel); (3) screening for HCP and parents (no neonates).

Our unit adopted a universal screening for HCPs, parents, and neonates.

\section{Results}

During the pandemic period (from February 21 to April 21, 2020), we admitted 65 neonates and 114 parents. A total of 954 nasopharyngeal swabs were collected from 226 individuals. Two parents and three HCPs tested positive and were kept isolated for 14 days. They were all asymptomatic. Positive cases did not have severe infection and were treated at home. The absence from work of positive cases was 45 days in total, 2 weeks per positive HCP.

Table 1 summons up the costs sustained during the pandemic period (1st trimester 2020), and the two following trimesters of 2020, as compared to the same time-shifts in 2019. During the 2 nd and 3rd trimesters, we did not record any case among HCPs and parents. The 9-month costs for personal protective equipment and nasopharyngeal swabs doubled in 2020 vs 2019. However, while the unitary cost for NF swabs remained unchanged throughout the three trimesters of 2020, the number of PCR test for SARS-CoV-2 decreased during the last trimester, according to the lower R0, while the number of PPEs used increased from the 1 st to the 3rd trimester of 2020.

Standing the actual costs, we projected the estimated expense under the three different screening policies: (1) screening for HCP and neonates (no parents); (2) screening for neonates and parents (no personnel); (3) screening for HCP and parents (no neonates). The projection was conducted under four different R0 ranging from 0.5 to 4.5 . The interaction among parents, neonate, and healthcare personnel is displayed in Fig. 1.

We estimated that one HCP could have infected 9, 27, 54, and 81 contacts under four R0 scenarios $(0.5,1.5,3$, and 4.5), but the number of infected was capped to the maximum number of contacts per shift (14 HCPs and 4 parents); a parent could have infected $1.5,4.5,9$, and 13.5 contacts plus her/his own baby. The neonate estimated contagious rates were fixed at four (one nurse, one resident, one non-medical assistant, one attending) per each shift, independently from the R0 (Fig. 2a, b).

Cost of the prevention campaign, per shift, was 11.000 EUR (100EUR/week for PPE and alcoholic solution, over gown $+65.0 /$ swab including the 60 parents admitted and 14 HCPs per shift).

Figure $2 \mathrm{~b}$ depicts projected savings under different R0 scenarios and screening policies. Costs are represented by the PPE (fixed cost) and the nasopharyngeal swabs, while "savings" are costs of work days saved under different rates of contagious. Savings were estimated with respect to the number of expected contacts and the working day loss/HCP unit.
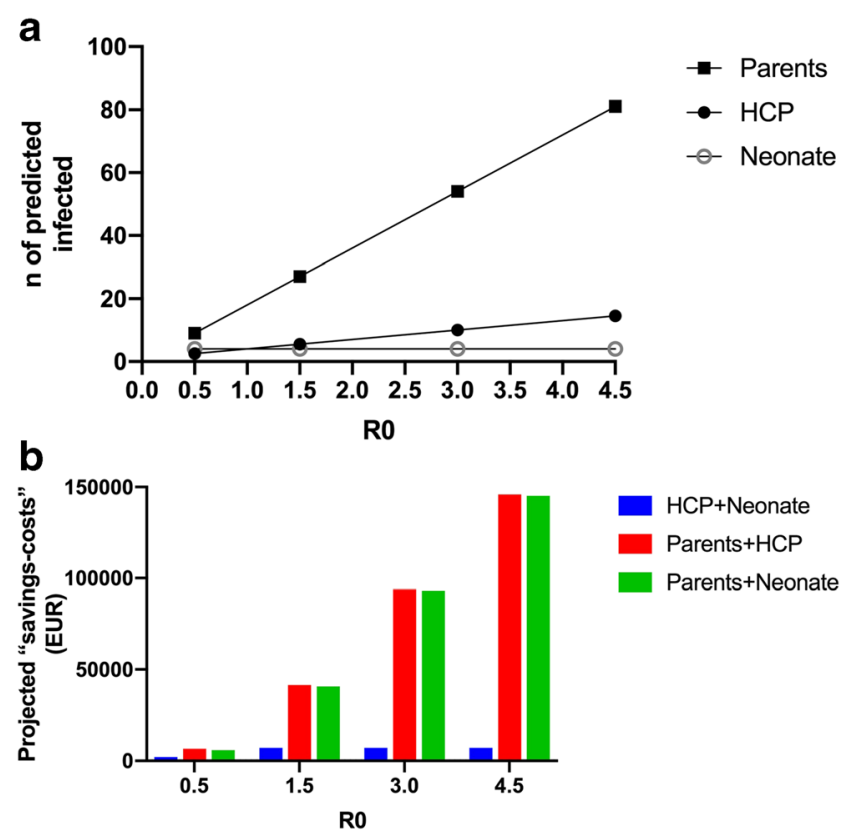

Fig. 2 a Number of predicted infected cases under different R0. b Projected savings-costs analysis according to the expected costs of lost work days in case of contagious

\section{Conclusion}

The pandemic screening measurements and the implementation of PPEs use in a NICU setting doubled the costs of management during the first 9 months of 2020 as compared to the same period of 2019. In spite of differential limitations observed out of the hospital setting, the costs for screening test remained unchanged over time in 2020 with a progressive increase in PPE use and associated expense. The measures were effective to prevent new in-hospital cases among the personnel and the parents regardless of the relative fluctuations of the R0 in north-eastern Italy.

While under $\mathrm{R} 0=0.5$ scenario we observed a minimal difference with the three screening strategies, it dramatically increased with $\mathrm{R} 0 \geq 1.5$. The adoption of parents' screening (with neonates or with HCPs), under an R0 $=4.5$ scenario, was associated with an expected saving of $\sim 140,000$. The estimated saving derives from the HCP work days that should have been replaced with additional units in case of infection.

Parents' screening was associated with the most costeffective saving scenario in the presence of $\mathrm{R} 0 \geq 1.5$, since parents have at least 4 contacts per day with HCPs plus their own baby, and interact with multiple shifts during the week. Therefore, while the number of potential infections is capped for HCP (whose shifts are unchanged over the month), the number of contacts due to infected parents can grow exponentially by involving multiple shifts even in the presence of HCP and neonate screening policies.

This cost analysis confirmed that a heightened surveillance in NICU, involving both HCP and parents, could save the 
highest personnel costs. Monitoring the health status of all the personnel and the parents has permitted to keep the environment safe, preventing the spread of the virus among staff and parents and avoiding any horizontal transmission between hospitalized infants. [6]

Even though such results cannot be generalized to other countries or to developing areas, due to different costs of personnel, sick-days policies, and screening costs, they provide a clear insight into the effectiveness of parents' screening in NICU setting, and preserve the ability of parents to visit their newborns in spite of a pandemic crisis. The cost estimates are strictly limited on regional policies for healthcare expenses, materials purchased, and testing facilities' capability.

Our NICU screening policy intensified the frequency of healthcare personnel and parents with respect to the general hospital policies, due to the specific features of our environment. [7] Therefore, our results cannot be generalized to the hospital as well as to other departments than NICU.

Our estimates largely minimize the impact of out-ofhospital infection of each of the involved actors and assume a capped number of contacts for HCPs and parents that is warrantied only under restrictive management personnel policies and within the specific regional contest the study has been conducted.

The lack of generalizability of such a cost-effectiveness analysis is, therefore, a major limitation of this report, but it provides a unique insight in a regional universally covered healthcare system and into the measurements in place to minimize the impact of the pandemic on the workflow in a NICU setting.

The rate of infection and the prevalence of the virus in the general population are key factors in evaluating the effectiveness of screening policies. The reduced benefits deriving from low $\mathrm{R} 0$ is suggestive for a flexible screening policy according to the regional $\mathrm{R} 0$ of the virus and calls for careful monitoring from local healthcare authorities of the population prevalence of the infection by large screening policies.

Supplementary Information The online version contains supplementary material available at https://doi.org/10.1007/s00431-020-03884-1.

Authors' Contributions AG designed the study analysis, run the analysis and drafted the manuscript. EL designed the study analysis, collected data and drafted the manuscript. MEC collected data and revised the manuscript draft. LB collected data and revised the manuscript draft. DT critically revised the data analysis and the draft. EB critically revised the data analysis and the draft. All the authors contributed and approved the final version of the current manuscript.
Data availability Data are available from the corresponding author upon request.

\section{Compliance with ethical standards}

Conflict of interest The authors declare that they have no conflict of interest.

Ethical approval This article does not contain any studies with human participants or animals performed by any of the authors.

Consent to participate N/A.

Consent for publication The authors consent to the manuscript publication.

Code availability N/A.

\section{References}

1. Cavicchiolo ME, Lolli E, Trevisanuto D, Baraldi E (2020) Managing a tertiary-level NICU in the time of COVID-19: lessons learned from a high-risk zone. Pediatr Pulmonol 2020, 55(6):-1310. https://doi. org/10.1002/ppul.24788

2. Buonsenso D, Costa S, Sanguinetti M, Cattani P, Posteraro B, Marchetti S, Carducci B, Lanzone A, Tamburrini E, Vento G, Valentini P (2020) Neonatal late onset infection with severe acute respiratory syndrome coronavirus 2 . Am J Perinatol 2020, 37(8):872. https://doi.org/10.1055/s-0040-1710541

3. Shalish W, Lakshminrusimha S, Manzoni P et al (2020) COVID-19 and neonatal respiratory care: current evidence and practical approach. Am J Perinatol. 05/02/2020. https://doi.org/10.1055/s0040-1710522

4. Vivanti AJ, Vauloup-Fellous C, Prevot S et al (2020) Transplacental transmission of SARS-CoV-2 infection. OriginalPaper. Nat Commun. 2020-07-14 11(1):1-7. https://doi.org/10.1038/s41467020-17436-6

5. CDCgov. Information for healthcare professionals about coronavirus (COVID-19) | CDC. 2020-07-07T09:18:55Z 2020

6. JPd W, Luca DD, Tingay DG (2020) COVID-19 surveillance for all newborns at the NICU; conditio sine qua non? EditorialNotes. Eur J Pediatr 2020-08-12:1-3. https://doi.org/10.1007/s00431-020-03773-7

7. Donà $\mathrm{D}$, Giaquinto $\mathrm{C}$, Baraldi $\mathrm{E}$, Biffi $\mathrm{A}$, Gamba $\mathrm{P}$, Saieva $\mathrm{AM}$, Antoniello L, Costenaro P, Masiero S, Sainati L, Da Dalt L, Perilongo G (2020) COVID-19 pandemic: perspective of an Italian tertiary care pediatric center. Healthcare (Basel) 8(3):311. https://doi. org/10.3390/healthcare 8030311

Publisher's note Springer Nature remains neutral with regard to jurisdictional claims in published maps and institutional affiliations. 